\title{
A Novel Circular RNA circTADA2A Promotes Proliferation And Metastasis Of Ovarian Cancer Through Sponging miR-203
}

\section{Aihong Wang}

The First Affliated Hospital, College of Clinical Medicine, Henan University of Science and Technology

\section{Canhui Jin}

The First Affiliated Hospital, College of Clinical Medicine, Henan University of Science and Technology

\section{Ying Wang}

The First Affiliated Hospital, College of Clinical Medicine, Henan University of Science and Technology Juanjuan Yu

The First Affiliated Hospital, College of Clinical Medicine, Henan University of Science and Technology

\section{Ruifang Wang}

The First Affiliated Hospital, College of Clinical Medicine, Henan University of Science and Technology

\section{Xiaoyu Tian ( $\nabla$ xiaoyu_tian123@126.com )}

Department of Gynecologic and Obstetrics, The First Affiliated Hospital, College of Clinical Medicine, Henan University of Science and Technology, NO.24, Jinhua Road, Luoyang 471000, China https://orcid.org/0000-0001-5667-4547

\section{Research}

Keywords: circTADA2A, OC, miR-203, SMAD1, ceRNA

Posted Date: January 13th, 2021

DOl: https://doi.org/10.21203/rs.3.rs-143578/v1

License: (a) (1) This work is licensed under a Creative Commons Attribution 4.0 International License. Read Full License 


\section{Abstract}

\section{Background}

Circular RNAs (circRNAs), a new type of non-coding RNA, have been demonstrated to play critical roles in the progression of various of malignant cancers. In the present study, we identified a circRNA termed as circTADA2A which was hypothesized that may be significantly up-regulated in $O C$ tissues and cell lines.

\section{Results}

The results revealed that ectopic expression of circTADA2A promoted the cell proliferation, migration, invasion and colony formation ability of OC cells. Constantly, silencing of circTADA2A inhibited those of OC cells. Furthermore, we identified that circTADA2A was able to target miR-203 in OC cell. MiR-203 was able to reverse the oncogenic effect of circTADA2A on proliferation, migration and metastasis of OC cells through targeting SMAD1.

\section{Conclusions}

We reported that circTADA2A served as a competing endogenous RNA (ceRNA) to sponge miR-203 and blocked its regulation of SMAD1. These findings provide insights into $\mathrm{OC}$ progression and also potential new targets for diagnose or treatment of OC.

\section{Background}

Ovarian cancer $(\mathrm{OC})$ is one of the leading causes of cancer-related death among females in China and around the world [1-3]. Despite the great progress in the early diagnosis and therapeutic methods, the prognosis of $\mathrm{OC}$ is still poor. Due to the lack of early diagnostic biomarkers, $\mathrm{OC}$ is always found in advanced stage $[1,4,5]$. In this regard, it is of great importance to develop novel molecular or strategies to achieve the purpose of early diagnosis and also improve the overall prognosis of OC patients.

Circular RNAs (circRNAs) are an innovative class of the noncoding RNAs, characterized by a covalently closed loop without 5' to 3' polyadenylated tails[6, 7], which are developed by back splicing events. Currently, circRNAs have been illustrated as highly conserved and abundant with high stability across various species $[8,9]$. With numerous investigations underway, circRNA was observed as differentially expressed in various cancers[6, 9-12]. Also, they were indicated as being involved in several physiological and pathophysiological mechanisms, for instance, modulating alternative splicing, which serves as microRNA (miRNA) sponges, together with regulating the protein-RNA interactions, accordingly regulating the cell phenotype[6, 10].

MicroRNAs (miRNAs) are small endogenous noncoding capable of regulating the core cell process by means of binding to the specific requirements of the $3^{\prime}$ untranslated region (3'UTR) of target genes $[12,13]$. This binding has the potential to inhibit protein translation or induce the degradation of target mRNAs. 
CircRNAs primarily sponge miRNAs to exert an impact on not only the gene expression but also biological functions[10, 12, 14].

In the current research work, we figured out an up-regulated circular RNA hsa_circ_0043278 (circTADA2A) in $\mathrm{OC}$, together with finding its up-regulated expression level in both $\mathrm{OC}$ tissues and cell lines. The loss and gain of function studies were carried out for the determination of the function of circTADA2A in tumorigenesis of OC. Mechanistically, we not only predicted but also verified the target miRNA of circTADA2A, which is miR-203, in addition to further elucidating the underlying mechanism of circTADA2A in OC. To summarize, circTADA2A works as an oncogene in OC by sponging milR-203, besides being likely to constitute a potential therapeutic target for OC.

\section{Results}

The expression of circTADA2A was upregulated in OC tissues and cells

Aimed at exploring the role of circRNAs in the progression of OC, we first performed RNA sequencing. Figure $1 \mathrm{~A}$ showed the most significantly up-regulated and down-regulated circRNAs, among which we selected has_circRNA_102049 termed as circTADA2A. As we know, circRNAs have much more stability for their looped structure. Accordingly, for the purpose of confirming the stability of circTADA2A, RNase $R$ was employed in the experiments. As the results suggested, following the RNase R treatment, the linear forms of TADA2A showed an evident decline while no significant change was observed in the level of circTADA2A, indicating the stability of circTADA2A (Figure 1B). Also, OC cells were treated with Actinomycin $D$ in order to inhibit the transcription after which the half-life of circTADA2A was assessed (Figure 1C). The results shed light on a longer half-life of circTADA2A as compared with that of TADA2A mRNA. Besides that, a specific probe was designed and made to carry out the FISH experiment. Figure 1D demonstrates that circTADA2A located primarily in cytoplasma. We detected the expression level of circTADA2A with the use of qPCR. The results illustrated that circTADA2A was remarkably up-regulated in the $O C$ tissues and cells (Figure 1E, F).

\section{CircTADA2A promoted the proliferation and migration of OC cells}

Owing to the modified expression in OC tissues and cell lines, we speculated that circTADA2A plays quite a crucial role in the progression of OC. Thus, the gain and loss of function studies were carried out with the use of overexpressing and shRNA vectors for circTADA2A. The qPCR analysis verified the overexpressing or knock down efficient of the overexpressing and shRNA vectors for circTADA2A, correspondingly (Figure 2A). Subsequently, as the MTT results suggested, the circTADA2A overexpression promoted cell proliferation while the circTADA2A knock down inhibited that of OC cells (Figure 2B). Colony formation was further carried out. As expected, the circTADA2A overexpression augmented the colony formation capability, while silencing of circTADA2A lowered it in OC cells (Figure 2C). Moreover, transwell assay and wound healing were carried out for the evaluation of the invasion and migration ability of OC cells. As the results suggested, circTADA2A overexpression augmented the invaded cell number in transwell assay (Figure 2D) as well as the migrated distance in wound healing assay (Figure 
$2 \mathrm{E})$. Contrastingly, the silencing of circTADA2A lowered that capability of OC cells as compared with the shRNA control group.

\section{CircTADA2A exerts its function by sponging miR-203}

It is well known that circRNAs sponge miRNAs for regulating the downstream gene expression for the purpose of participating in regulating the cell physiology mechanism. We carried out the bioinformatics analysis software circinteractome, aimed at predicting the potential miRNA target of circTADA2A, followed by selecting miR-203. Figure 3A demonstrated the complementary base sequences between circTADA2A and miR-203. The luciferase activity assay was carried out for the verification of the binding of circTADA2A and miR-203. We discovered the fact that miR-203 lowered the luciferase activities of the wild-type reporter for circTADA2A but not that of the mutant-type reporter, confirming miR-203 as a sponge target of circTADA2A (Figure 3B). Subsequent to that, we carried out the circTADA2A overexpressing and shRNA vector transfection. Interestingly, circTADA2A overexpression lowered the level of miR-203 while the circTADA2A knock down augmented the same (Figure 3C). In addition, Pearson analysis was used for evaluating the relationship between circTADA2A and miR-203; as the results suggested, there was a significant negative relationship existing between circTADA2A and miR-203 (Figure 3D). The RNA pull-down was further performed for the purpose of confirming the binding of circTADA2A and miR-203. By means of the results, we figured it out that the enrichment of miR-203 was substantially higher in the circTADA2A probe pull down product in comparison with that of the control probe (Figure 3E). Accordingly, more circTADA2A was captured by the biotin-labelled miR-203 groups as compared with the control probe (Figure 3F). These findings suggested miR-203 as a sponge targets of circTADA2A in OC cells.

\section{MiR-203 reversed the function of circTADA2A in OC cells}

Being a target of circTADA2A, we predicted that miR-203 had been involvement in the biological mechanism of OC cells as well. We carried out the miR-203 and circTADA2A overexpressing vector transfection. Co-transfection with miR-203 and circTADA2A overexpressing vector apparently augmented the level of miR-203 in comparison with circTADA2A overexpressing group (Figure 4A). By means of the function studies, we, at first, discovered that miR-203 reversed not only the cell proliferation (Figure 4 B), but also the colony formation ability (Figure 4C) invitation (Figure 4D) as well as migration (Figure 4E, F) of OC cells. Together with that, circTADA2A reversed these functions of miR-203 partially, further indicating the relationship existing between circTADA2A and miR-203.

\section{MiR-203 target SMAD1 in OC cells}

MiRDB, DIANA and Targetscan databases were used to predict the targets of miR-203, among which SMAD1 was selected as a potential one for its existence in all the three databases (Figure $5 \mathrm{~A}$ ). Figure $5 \mathrm{~B}$ showed the target region between miR-203 and SMAD1. Luciferase activity assay in both SKOV-3 and OVCAR-3 cells were carried out. As indicated by findings, miR-203 evidently down-regulated the luciferase activity co-transfected with pGL3-3'UTR of SMAD1, but not the pGL3-3'UTR-mut (Figure 5 C). RNA-pull 
down experiments were carried out, the results indicated that miR-203 directly target SMAD1 (Figure 5D). In addition, qPCR and western blot analysis further confirmed that miR-203 overexpression decreased the level of SMAD1 while knock down of miR-203 increased that of SMAD1 (Figure $5 \mathrm{E}, \mathrm{F}$ ). Pearson analysis revealed a negative relationship between miR-203 and SMAD1 (Figure 5G) which further verified the target relation between miR-230 and SMAD1. In order to confirm the ceRNA relation between circTADA2A and $S M A D 1$, we evaluated whether circTADA2A involved in the regulation of SMAD1 expression. The results verified our speculation. CircTADA2A notably promoted SMAD1 expression (Figure 5H) and Pearson analysis indicated a positive relation between circTADA2A and SMAD1 which further indicated their relationship (Figure 5l).

\section{CircTADA2A regulated AKT/GSK3 $\beta$ signal pathway and EMT proteins expression}

As SMAD1 was confirmed to be the target of miR-203, we focused on the downstream gene which may be regulated by SMAD1. Western blot was carried out to evaluate the specific protein expression. As figure 6 $A$ and $B$ revealed. CircTADA2A significantly promoted expression level of SMAD1, N-cadherin, Vimentin, MMP-9 along with the phosphorylation of AKT and GSK3 $\beta$. Meantime, circTADA2A inhibited the level of E-cadherin. These regulatory effect of circTADA2A was reversed by both miR-203 expression and knock down of SAMD1 indicating the target relation between circTADA2A and miR-203 along with the ceRNA relationship between circTADA2A and SMAD2.

\section{CircTADA2A promoted the growth of OC cells in vivo}

Thereafter, we carried out the in vivo study, aimed at further investigating the biological function of circTADA2A in the progression of OC. Stable SKOV-3 cell line was established with the transfection of shcircTADA2A or circTADA2A vectors along with their negative control, correspondingly. Both the size and weight of the tumours in the circTADA2A were observed as higher in comparison with the control tumours. Contrarily, the circTADA2A deletion lowered the tumour size and weight of tumours in comparison with the control group (Figure 7A-C). IHC assay, detecting the expression of ki67, the proliferation biomarker, was also carried out. In accordance with the expectations, circTADA2A overexpression promoted the expression of ki67 and SMAD2 while the knock down of circTADA2A inhibited that of ki67 and SMAD2 (Figure 7D).

\section{Discussion}

CircRNAs have been fully documented as playing critical roles in the cellular functions, for instance, proliferation, apoptosis, differentiation, and metabolism[6, 11]. In the current research work, we figured out an innovative circRNA, termed as circTADA2A. The stability of circTADA2A was confirmed by its stable expression subjected to the Rnase $\mathrm{R}$ digestion. To the best of our understanding, this is the first study dealing with the circTADA2A emphasizing its modified expression as well as biological impact. The up-regulated expression and stability of circTADA2A make it a potential biomarker as a diagnose and therapeutic target. 
It is well known that circRNAs mainly act as the sponges of miRNAs, thus block their regulatory effect on the downstream gene expression. Among them, miR-203 has been extensively investigated and proved to play crucial role in the cellular differentiation and development. Several research works shed light on the anti-cancer function of miR-203 in OC. It was confirmed to inhibit proliferation and metastasis of OC cells, meantime, increase apoptosis of OC cells[15-17]. Recently, miRNAs were found to be sponged by circRNAs and IncRNAs that further block their regulatory effects on cell process. For instance, CircAGFG1 sponges miR-203 to promote EMT and metastasis of non-small-cell lung cancer by upregulating ZNF281 expression[18]. HCP5 is a SMAD3-responsive long non-coding RNA that promotes lung adenocarcinoma metastasis via miR-203/SNAI axis[19]. However, the relationship between cicRNA and miR-203 in OC has not yet been dicussed. In the present study, we predicted and verified that miR-203 was sponged by circTADA2A which could block its anti-cancer role. To further elucidate the mechanism of miR-203, we predicted the target genes through three prediction software including Targetscan, miRDB and DIANA and get 564 genes exist in all 3 datasets. Among them, SMAD1 was selected and verified as the target of miR203.

SMA and mother against decapentaplegic (MAD)-related proteins (SMADs) are intracellular components of TGF- $\beta$ signalling pathway. containing eight members as SMAD1-Smad8 respectively[20-23]. SMAD1 is a critical inducer of the EMT process. SMAD1/Akt/GSK3 $\beta$ signalling pathway regulated by snail altered Nanog status during EMT[24]. Another EMT transcription factor Twist1 also plays the role as a downstream factor of SMAD1[25]. In the present research, we predicted and confirmed that miR-203 target SMAD1 in SKOV-3 and OVCAR-3 cell. Due to the critical role of SMAD1 in EMT, we thus evaluated the EMT proteins such as E-cadherin, Vimentin, MMP-9 and N-cadherin and found that circTADA2A protmoted SMAD1 expression along with N-cadherin, Vimentin, MMP-9 expression. This effect of circTADA2A can be reversed by both miR-203 overexpression and knock down of SMAD1 indicating the ceRNA relation between circTADA2A and SMAD1. However, whether SMAD1 directly regulated EMT proteins expression or via other key molecular remain not elucidated. SMAD1 was reported to be involved in the AKT signal pathway. For instance, SMAD-PI3K-Akt-mTOR pathway mediates BMP-7 polarization of monocytes into M2 macrophages. It was figured out that SMAD1 was capable of promoting the phosphorylation of AKT and GSK3 3 . We noticed that AKT was also demonstrated to regulate SMAD1 expression. AKT is involved in the cell proliferation especially in cancer cells[26, 27]. Whether there exist a negative loop regulation mechanism between AKT and SMAD1 remain unclear which is on our further exploration plan.

In the current research work, circTADA2A was identified as a novel circRNA which play a oncogenetic role in OC. MiR-203 was determined to be the target miRNA of circTADA2A. Interestingly, a recent study also figured out the effect of circTADA2A and miR-203a in osteosarcoma progression. CircTADA2A promote osteosarcoma progression through sponging miR-230a and alter CREB3 expression[28]. Aimed at extending the understanding of circTADA2A and miR-203 in the develop of human cancer, we predicted the target gene of miR-203, besides finding SMAD1 as a potential one which have a close association with the EMT in human cancers and has not reported before. CircTADA2A/miR-203/SMAD1 signal axis 
was figure out which is likely to be involved in the regulation of cell phenotype including proliferation and metastasis of $\mathrm{OC}$ cells.

\section{Conclusions}

To conclude, our findings shed light on the fact that circTADA2A promotes the malignant behaviour of OC by means of sponging miR-203, which indicates its potentiality to be a diagnostic or therapeutic target of OC.

\section{Methods And Materials}

\section{Patients}

A total number of 25 paired $\mathrm{OC}$ and the adjacent tissues were collected from The People's Hospital of Linan City between May 2016 and Nov 2016. The samples were stored in liquid nitrogen immediately after surgery. This study has been proved by the ethical committee of The People's Hospital of Linan City and written informed consent was obtained from each patient.

RNA-seq analysis

The total RNAs were extracted from $3 \mathrm{OC}$ tissue and 3 normal tissue by TRIzol (Invitrogen). The tissue samples were then sent to Vazyme Biotech (Nanjing, China) for the sequencing analysis.

\section{Cell culture}

Ovarian cancer (OC) lines (SKOV-3, Caov-3, OVCAR-3 and HO-8910) as well as normal ovarian epithelial cell line (IOSE80) were obtained from the ATCC (Manassas, VA, USA). OC cells were cultured in RPMI Medium 1640(Gibco, CA, USA) and NHBE was cultured in Dulbecco's Modifed Eagle's Medium (DMEM) (Gibco, CA, USA) all containing 10\% FBS (fetal bovine serum, Gibco, CA, USA) supplemented with 100 $\mathrm{U} \cdot \mathrm{mL}-1$ penicillin, and $100 \mathrm{mg} \cdot \mathrm{mL}-1$ streptomycin (Invitrogen, CA, USA) in humid conditions with $5 \%$ $\mathrm{CO} 2$ at $37^{\circ} \mathrm{C}$.

\section{Cell transfection}

MiR-203 inhibitor and inhibitor control were synthesized by GenePharma (Shanghai, China). Overexpressing vector for CircTADA2A and pre-miR-203, control vector, short hairpin RNA (shRNA) targeting circTADA2A and its negative control were purchased from Ribobio (Guangzhou, China). Cells were seeded on 6-well plates at the density of $8 \times 10^{5}$, followed by getting cultured in RPMI- 1640 without FBS at a temperature of $37^{\circ} \mathrm{C}$ for $12 \mathrm{~h}$ prior to the transfection. Cell transfection and co-transfection were carried out with the use of Lipofectamine 2000 (Invitrogen, CA, USA) in accordance with the manufacturer's instructions. $6 \mathrm{~h}$ following the transfection, the cell culture medium was replaced with RPMI-1640 medium, which was supplemented with $10 \%$ FBS. 
$24 \mathrm{~h}$ following the transfection, cells were collected and resuspended in culture medium, followed by seeding on 96 -well plates at a density of $1 \times 10^{4}$ cells/well. Moreover, $10 \mu$ l CCK-8 solution was added to each well and incubated for another 2 hours at a temperature of $37^{\circ} \mathrm{C}$. Absorbance at $480 \mathrm{~nm}$ was detected using a microplate Reader (Biorad, CA, USA). Each sample was performed for a minimum of 3 times.

\section{Transwell assay}

Subsequent to the transfection, $1 \times 10^{5}$ of cells suspended in $200 \mu \mathrm{L}$ RPMI- 1640 medium were seeded onto the upper transwell chamber (Corning, NY, USA). Following 24 hours of incubation at $37^{\circ} \mathrm{C}$, the cells on the surfaces of lower chambers were fixed using $20 \%$ methanol followed by staining with $1 \%$ crystal violet (Beyotime, Shanghai, China). Eventually, the cell colonies were both photographed and counted.

\section{Wound healing assay}

Subsequent to the transfection, the cells were seeded onto 6 well plates followed by culturing in serum free medium for a period of 24 hours. Thereafter, cell monolayers were wounded using a 10 $\mu$ l pipette tip. After that, cells were replaced with fresh medium and cultured at $37^{\circ} \mathrm{C}$. The pictures were taken 48 after the wounding for the purpose of determining the wound-closing procedure.

\section{Colony-formation assay}

Following the transfection, the cells were seeded in 12 well plates at the density of 100/well. Subsequent to the incubation for 2 weeks, cells were fixed in $10 \%$ formaldehyde, together with staining with $1 \%$ crystal violet (Beyotime, Shanghai, China). Images were photographed under a microscope (Leica, Germany), besides counting the colonies that contained more than 50 cells.

\section{Xenograft model}

SKOV-3 cells were harvested and resuspended in RPMI-1640 medium. The nude mice were injected with a total number of $3 \times 10^{6} / 100 \mu \mathrm{L}$ cells subcutaneously at the posterior flank. In addition, tumour size was monitored through the measurement of the length $(\mathrm{L})$ and width $(W)$ using callipers every 3 days. Following a period of 28 days, the tumours were excised out from the sacrificed mice and weighed.

Real-time PCR

Total RNA was extracted with the use of TRIzol reagent (Invitrogen, CA, USA) in accordance with the manufacturer's instructions. Afterwards, RNA was reverse transcribed to cDNA with the help of a PrimeScript RT Reagent kit (Takara, Dalian, China). The quantitative real-time PCR (qRT-PCR) analysis was carried out with the help of a SYBR-Green PCR Master Mix (Thermo Fisher, MA, USA) in a 7900HT PCR System (ABI, CA, USA). The use of GAPDH and U6 was made as internal controls for the mRNA and 
miRNA analysis, correspondingly. The relative expression levels were assessed with the use of the $2-\Delta \Delta \mathrm{Ct}$ methodology. All of the reactions were carried out in triplicate.

\section{Western blot}

The total protein was extracted from cells with the help of the RIPA lysis buffer (Beyotime, Shanghai, China). The protein concentration was detected in a nanodrop system (Thermo Scientific, MA, USA). 40ug protein was separated by $10 \%$ SDS-PAGE, followed by transferring to polyvinylidene difluoride membranes (Millipore, CA, USA). Subsequently, the blots were blocked using $5 \%$ non-fat milk for a period of 2 hours at the room temperature followed by the incubation at $4^{\circ} \mathrm{C}$ overnight with primary antibodies. Thereafter, the incubation of membranes was performed with a corresponding horseradish peroxidaseconjugated secondary antibody for 4 hours at the room temperature. The bands were detected with the use of an enhanced chemiluminescence solution (Pierce, MA, USA), together with imaging using the FluorChem imaging system (Alpha Innotech, San Leandro, CA, USA). The use of GAPDH was made as the internal control. All of the reactions were carried out in triplicate.

\section{Fluorescence in situ hybridization (FISH)}

Alexa Fluor 555-labeled circTADA2A probes were designed and synthesized by RiboBio (Guangzhou, China). FISH experiment was carried out using a fluorescent in Situ Hybridization Kit (RiboBio, Guangzhou, China). $1 \times 10^{5}$ cells were seeded onto the autoclaved glass slides and cultured for a period of 24 hours. Subsequent to fixing with $4 \%$ paraformaldehyde for 20 minutes, followed by the permeabilization with $0.5 \%$ Triton $\mathrm{X}-100$ for 10 minutes, the cells were cultured at $37^{\circ} \mathrm{C}$ overnight. Eventually, the incubation of slides was carried out with DAPI for the purpose of staining the cell nuclear and observed under a fluorescence microscope (Leica, Wetzlar, Germany).

\section{RNA pull down}

The biotin labelled circTADA2A as well as miR-203 probes along with theri control probe were synthesized by Sangon Biotech (Shanghai, China). In addition, the probe-coated beads were generated through the coincubation of the probe with the streptavidin-coated beads (Invitrogen, CA, USA) at $25^{\circ} \mathrm{C}$ for 2 hours. Cells were gathered and incubated with specific probes overnight at $4^{\circ} \mathrm{C}$. Following that, the beads were eluted and the complex was purified with TRIzol (Takara, Dalian, China). Afterwards, the abundance of both circTADA2A and miR-203 was analysed by the qRT-PCR.

$I H C$

The tumour tissues were fixed in $4 \%$ paraformaldehyde for 24 hours, followed by dehydrating in a graded alcohol series and embedding in paraffin, followed by cutting into $5 \mu \mathrm{m}$ sections. The sections were deparaffinised, rehydrated with a graded alcohol series and then incubated in $96^{\circ} \mathrm{C}$ with $0.01 \mathrm{~mol} / \mathrm{I}$ sodium citrate buffer for the antigen retrieval. Following the incubation in $5 \% \mathrm{H}_{2} \mathrm{O}_{2}$ for a period of 2 hours, the sections were incubated using primary antibodies including ki67 and SMAD1 (Abcam, England) 
overnight at $4^{\circ} \mathrm{C}$. Immunostaining was carried out with the use of streptavidin-peroxidase and diaminobenzidinef (DAB) following the manufacturer's instructions (Beyotime, Shanghai, China). Eventually, the sections were not only observed under a fluorescence microscope (Leica, Wetzlar, Germany) but also imaged.

\section{Luciferase reporter assay}

Cells were transfected with miR-203 mimics or mimic control and co-transfected with pGL3 reporter vectors (Promega, CA, USA) that contained the wild-type ( $\mathrm{Wt}$ ) or mutated (mut) potential binding sequence of circTADA2A as well as SMAD1. 48 hours following the transfection, cells were gathered and analysed with the help of a Dual-Luciferase Reporter Assay kit (Promega, CA, USA). Luciferase activity was detected through the use of a GloMax fluorescence reader (Promega, CA, USA). Renilla luciferase activities were put to use as an internal control. Each of the assays was carried out in a minimum of triplicate.

\section{Statistical analysis}

All data are presented as the mean $s \pm$ standard deviation (SD). The statistical analyses were performed using SPSS 20 software (Abbott Laboratories, Chicago, IL, USA). Data were analysed with one-way ANOVA and Student's test. $p<0.05$ was considered to be statistically significant.

\section{List Of Abbreviations}

\begin{tabular}{|ll|}
\hline OC & ovarian cancer \\
\hline CircRNAs & circular RNAs \\
\hline miRNAs & microRNAs \\
\hline shRNA & short hairpin RNA \\
\hline qRT-PCR & quantitative real-time PCR \\
\hline ceRNA & competing endogenous RNA \\
\hline L & length \\
\hline W & width \\
\hline FISH & Fluorescence in situ hybridization \\
\hline
\end{tabular}

\section{Declarations}

Ethics approval and consent to participate

This study was approved by the ethical committee of The People's Hospital of Linan City and written informed consent was obtained from each patient. 
Consent to participate

Not applicable.

Availability of data and materials

Not applicable.

Competing interests

The authors declare that they have no competing interests.

Funding

Not applicable.

Authors' contributions

Aihong Wang developed hypotheses and designed experiments. Canhui Jin performed experiments and wrote the manuscript. Ying Wang contributed to the collection and analysis of patient samples. Juanjuan Yu helped to draft the manuscript. Ruifang Wang analysed data and collected the clinical data.Xiaoyu Tian is mainly responsible for the design of the experiment, the interpretation of the data, the critical review of the paper and the contribution of the article.

\section{Acknowledgements}

This research did not receive any specific grant from funding agencies in the public, commercial, or notfor-profit sectors.

\section{References}

1. Alvarez Secord A, Bell Burdett K, Owzar K, Tritchler D, Sibley AB, Liu Y, Starr MD, Brady JC, Lankes HA, Hurwitz HI, Mannel RS, Tewari KS, O'Malley DM, Gray H, Bakkum-Gamez JN, Fujiwara K, Boente M, Deng W, Burger RA, Birrer MJ, Nixon AB. Predictive Blood-Based Biomarkers in Patients with Epithelial Ovarian Cancer Treated with Carboplatin and Paclitaxel with or without Bevacizumab: Results from GOG-0218. Clin Cancer Res. 2020;26(6):1288-1296.

2. Wang K, Zhu G, Bao S, Chen S. Long Non-Coding RNA LINC00511 Mediates the Effects of ESR1 on Proliferation and Invasion of Ovarian Cancer Through miR-424-5p and miR-370-5p. Cancer Manag Res. 2019;11:10807-10819.

3. Chen Q, Zhang ZH, Wang S, Lang JH. Circulating Cell-Free DNA or Circulating Tumor DNA in the Management of Ovarian and Endometrial Cancer. Onco Targets Ther. 2019;12:11517-11530.

4. Vuorinen RK, Luukkaala TH, Mäenpää JU. The influence of bevacizumab on the costs of ovarian cancer treatment in routine clinical practice. Acta Oncol. 2020;59(4):453-457. 
5. Montemorano L, Lightfoot MD, Bixel K. Role of Olaparib as Maintenance Treatment for Ovarian Cancer: The Evidence to Date. Onco Targets Ther. 2019;12:11497-11506.

6. Yu Q, Liu P, Han G, Xue X, Ma D. CircRNA circPDSS1 promotes bladder cancer by down-regulating miR-16. Biosci Rep. 2020;40(1):BSR20191961.

7. Li H, Shen S, Ruan X, Liu X, Zheng J, Liu Y, Yang C, Wang D, Liu L, Ma J, Ma T, Wang P, Cai H, Li Z, Zhao L, Xue Y. Biosynthetic CircRNA_001160 induced by PTBP1 regulates the permeability of BTB via the CircRNA_001160/miR-195-5p/ETV1 axis. Cell Death Dis. 2019;10(12):960.

8. Mao J, Li T, Fan D, Zhou H, Feng J, Liu L, Zhang C, Wang X. Abnormal expression of rno_circRNA_014900 and rno_circRNA_005442 induced by ketamine in the rat hippocampus. BMC Psychiatry. 2020;20(1):1.

9. Ding HX, Xu Q, Wang BG, Lv Z, Yuan Y. MetaDE-Based Analysis of circRNA Expression Profiles Involved in Gastric Cancer. Dig Dis Sci. 2020;65(10):2884-2895.

10. Yang SJ, Wang DD, Zhou SY, Zhang Q, Wang JY, Zhong SL, Zhang HD, Wang XY, Xia X, Chen W, Yang SY, Hu JH, Zhao JH, Tang JH. Identification of circRNA-miRNA networks for exploring an underlying prognosis strategy for breast cancer. Epigenomics. 2020;12(2):101-125.

11. Li Y, Zhang J, Pan S, Zhou J, Diao X, Liu S. CircRNA CDR1as knockdown inhibits progression of nonsmall-cell lung cancer by regulating miR-219a-5p/SOX5 axis. Thorac Cancer. 2020;11(3):537-548.

12. Yan Z, Xiao Y, Chen Y, Luo G. Screening and identification of epithelial-to-mesenchymal transitionrelated circRNA and miRNA in prostate cancer. Pathol Res Pract. 2020;216(2):152784.

13. Gao L, Yan SB, Yang J, Kong JL, Shi K, Ma FC, Huang LZ, Luo J, Yin SY, He RQ, Hu XH, Chen G. MiR182-5p and its target HOXA9 in non-small cell lung cancer: a clinical and in-silico exploration with the combination of RT-qPCR, miRNA-seq and miRNA-chip. BMC Med Genomics. 2020;13(1):3.

14. Yu H, Ma J, Chen J, Yang Y, Liang J, Liang Y. LncRNA LINC00461 Promotes Colorectal Cancer Progression via miRNA-323b-3p/NFIB Axis. Onco Targets Ther. 2019;12:11119-11129.

15. Wang B, Li X, Zhao G, Yan H, Dong P, Watari H, Sims M, Li W, Pfeffer LM, Guo Y, Yue J. miR-203 inhibits ovarian tumor metastasis by targeting BIRC5 and attenuating the TGF $\beta$ pathway. $J$ Exp Clin Cancer Res. 2018;37(1):235.

16. Liu HP, Zhang Y, Liu ZT, Qi H, Zheng XM, Qi LH, Wang JY. MiR-203 regulates proliferation and apoptosis of ovarian cancer cells by targeting SOCS3. Eur Rev Med Pharmacol Sci. 2019;23(21):9286-9294.

17. Wang N, Liang H, Zhou Y, Wang C, Zhang S, Pan Y, Wang Y, Yan X, Zhang J, Zhang CY, Zen K, Li D, Chen X. miR-203 suppresses the proliferation and migration and promotes the apoptosis of lung cancer cells by targeting SRC. PLoS One. 2014;9(8):e105570.

18. Xue YB, Ding MQ, Xue L, Luo JH. CircAGFG1 sponges miR-203 to promote EMT and metastasis of non-small-cell lung cancer by upregulating ZNF281 expression. Thorac Cancer. 2019;10(8):16921701.

19. Jiang L, Wang R, Fang L, Ge X, Chen L, Zhou M, Zhou Y, Xiong W, Hu Y, Tang X, Li G, Li Z. HCP5 is a SMAD3-responsive long non-coding RNA that promotes lung adenocarcinoma metastasis via miR- 
203/SNAI axis. Theranostics. 2019;9(9):2460-2474.

20. Caddy JC, Luoma LM, Berry FB. FOXC1 negatively regulates BMP-SMAD activity and Id1 expression during osteoblast differentiation. J Cell Biochem. 2020;121(5-6):3266-3277.

21. Gao Y, Zhang R, Dai S, Zhang X, Li X, Bai C. Role of TGF- $\beta$ /Smad Pathway in the Transcription of Pancreas-Specific Genes During Beta Cell Differentiation. Front Cell Dev Biol. 2019;7:351.

22. Sisto M, Lorusso L, Ingravallo G, Ribatti D, Lisi S. TGF 1 1-Smad canonical and -Erk noncanonical pathways participate in interleukin-17-induced epithelial-mesenchymal transition in Sjögren's syndrome. Lab Invest. 2020;100(6):824-836.

23. Aki S, Yoshioka K, Takuwa N, Takuwa Y. TGF $\beta$ receptor endocytosis and Smad signaling require synaptojanin1, PI3K-C2a-, and INPP4B-mediated phosphoinositide conversions. Mol Biol Cell. 2020;31(5):360-372.

24. Liu CW, Li CH, Peng YJ, Cheng YW, Chen HW, Liao PL, Kang JJ, Yeng MH. Snail regulates Nanog status during the epithelial-mesenchymal transition via the SMAD1/Akt/GSK3 $\beta$ signaling pathway in non-small-cell lung cancer. Oncotarget. 2014;5(11):3880-94.

25. Li A, Xia X, Yeh J, Kua H, Liu H, Mishina Y, Hao A, Li B. PDGF-AA promotes osteogenic differentiation and migration of mesenchymal stem cell by down-regulating PDGFRa and derepressing BMPSMAD1/5/8 signaling. PLoS One. 2014;9(12):e113785.

26. Yoshihara M, Kajiyama H, Yokoi A, Sugiyama M, Koya Y, Yamakita Y, Liu W, Nakamura K, Moriyama Y, Yasui H, Suzuki S, Yamamoto Y, Ricciardelli C, Nawa A, Shibata K, Kikkawa F. Ovarian cancerassociated mesothelial cells induce acquired platinum-resistance in peritoneal metastasis via the FN1/Akt signaling pathway. Int J Cancer. 2020;146(8):2268-2280.

27. Liu M, Qiu Y, Xue Z, Wu R, Li J, Niu X, Yuan J, Wang Y, Wu Q. Small extracellular vesicles derived from embryonic stem cells restore ovarian function of premature ovarian failure through PI3K/AKT signaling pathway. Stem Cell Res Ther. 2020;11(1):3.

28. Wu Y, Xie Z, Chen J, Chen J, Ni W, Ma Y, Huang K, Wang G, Wang J, Ma J, Shen S, Fan S. Circular RNA circTADA2A promotes osteosarcoma progression and metastasis by sponging miR-203a-3p and regulating CREB3 expression. Mol Cancer. 2019;18(1):73.

\section{Figures}


A

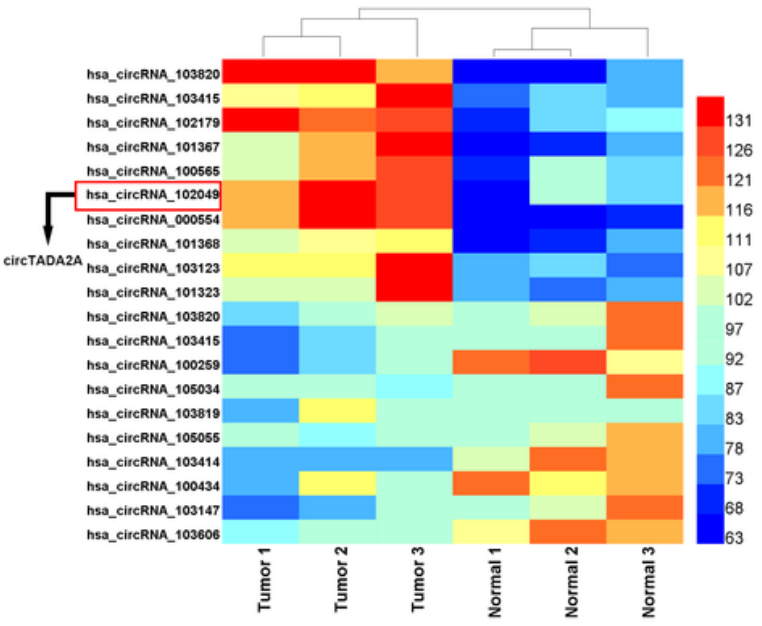

D

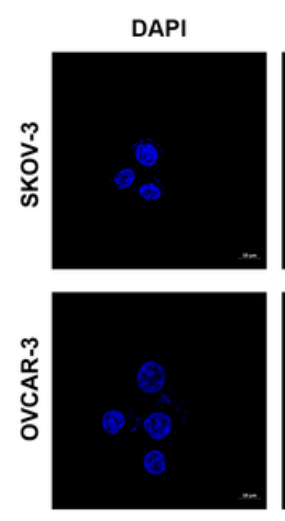

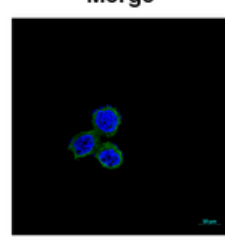
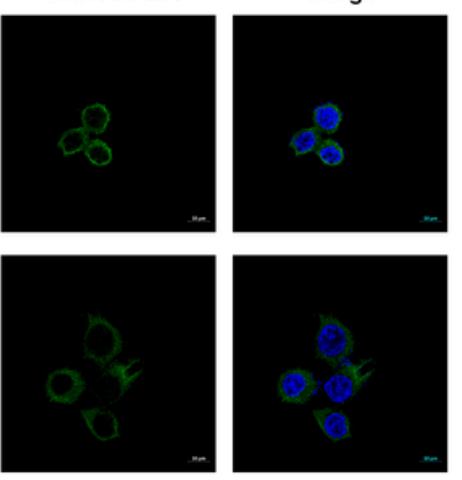

circTADA2A
B

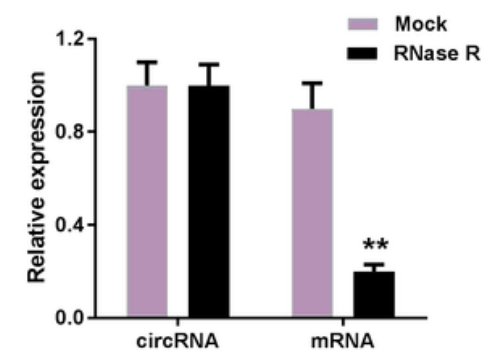

C

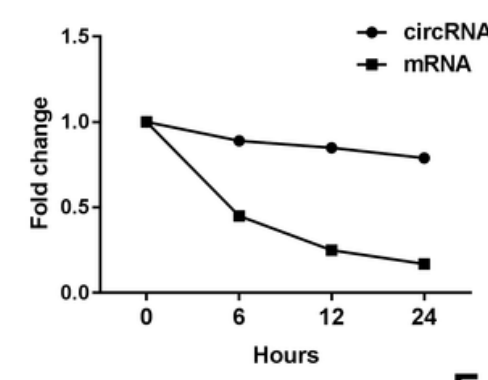

E

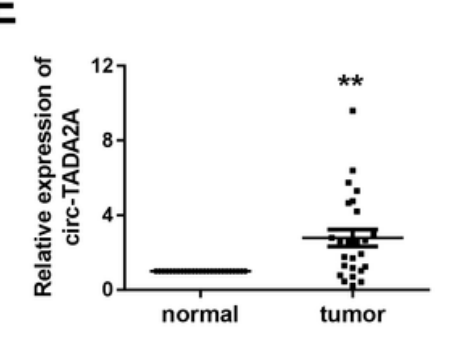

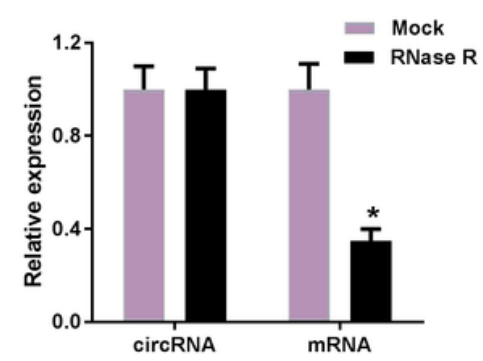

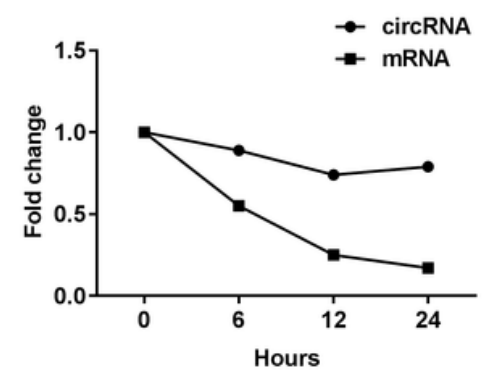

F

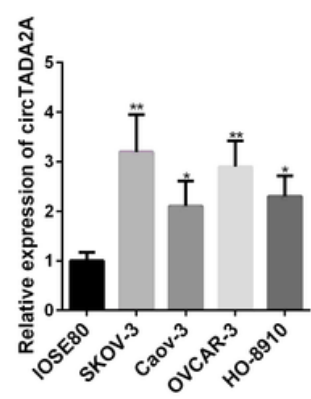

\section{Figure 1}

circTADA2A is upregulated in OC specimens and cell lines. (A) Analysis of GEO data (GEO) showed that circTADA2A was up-regulated in OC tissue and cell lines. (B) After treatment with Actinomycin D, the amount of circTADA2A and TADA2A mRNA from OC cells were evaluated using qPCR. (C) After RNase R treatment, the expression of linear mRNA of TADA2A and circTADA2A were detected using qPCR. (D) FISH assay with specific probe was performed to detect the expression and location of circTADA2A. Expression level of circTADA2A was evaluate by qPCR in OC tissues (E) and cell lines (F) compare to the adjacent normal tissues and ARPE19 cell. ${ }^{*} \mathrm{P}<0$ 05, compared with the mock, normal and NHBE group. $\star \star P<0$ 01, compared with the normal group. 
A

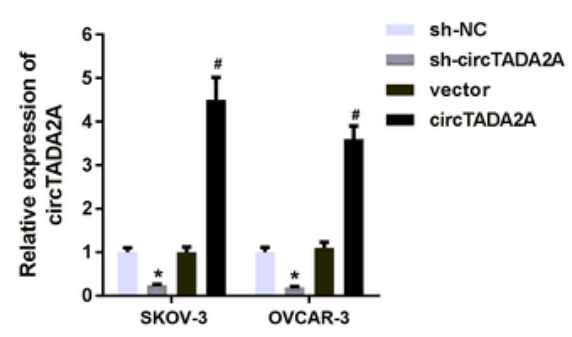

B

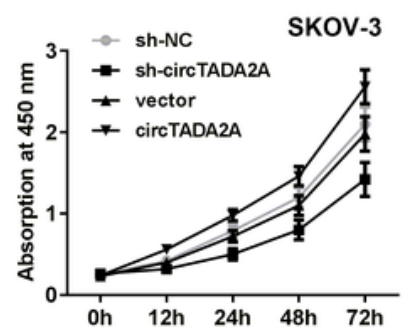

OVCAR-3

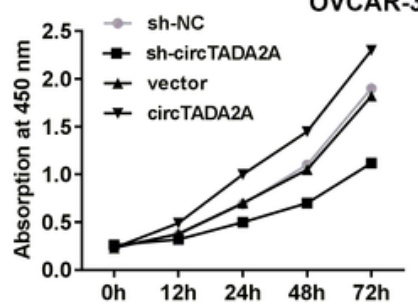

C

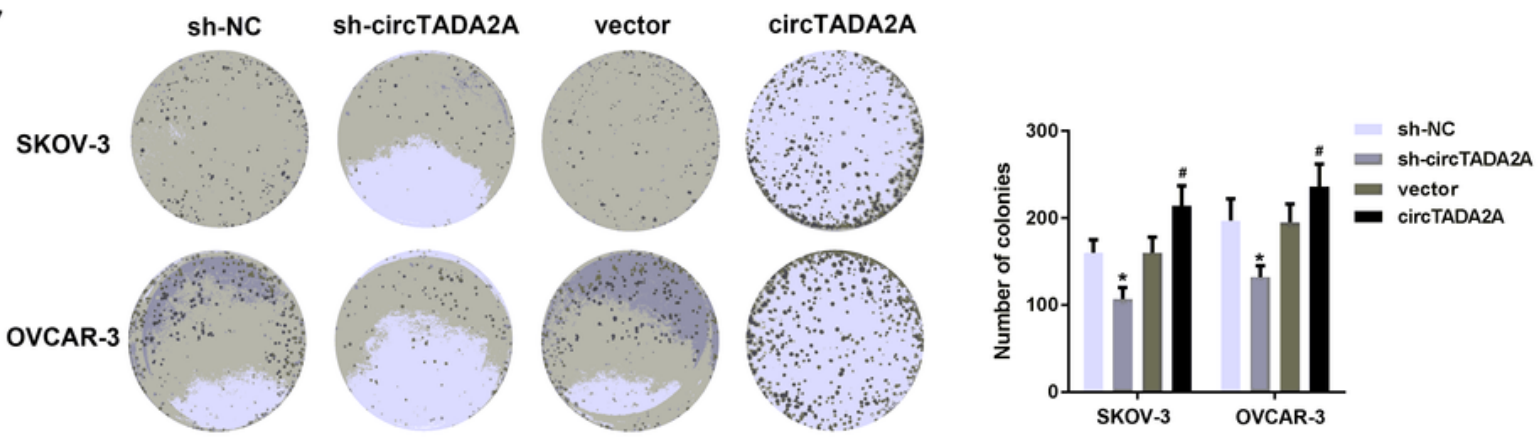

D

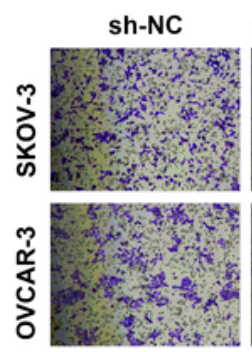

sh-circTADA2A

vector

circTADA2A
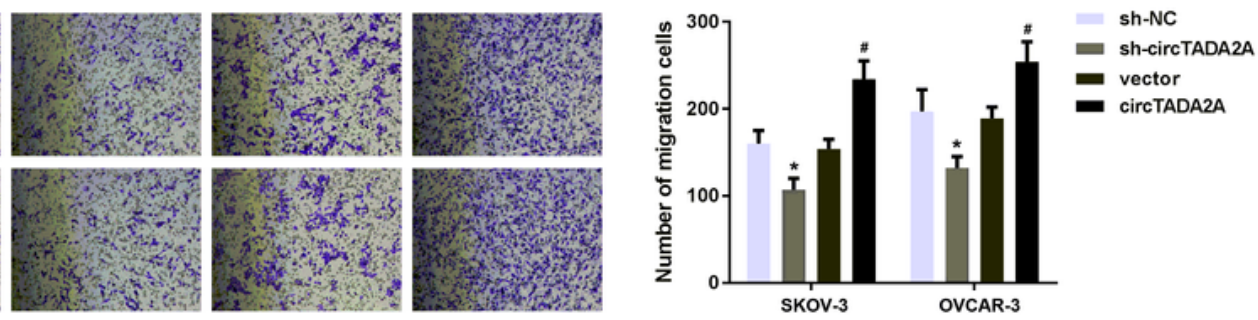

$\mathbf{E}$
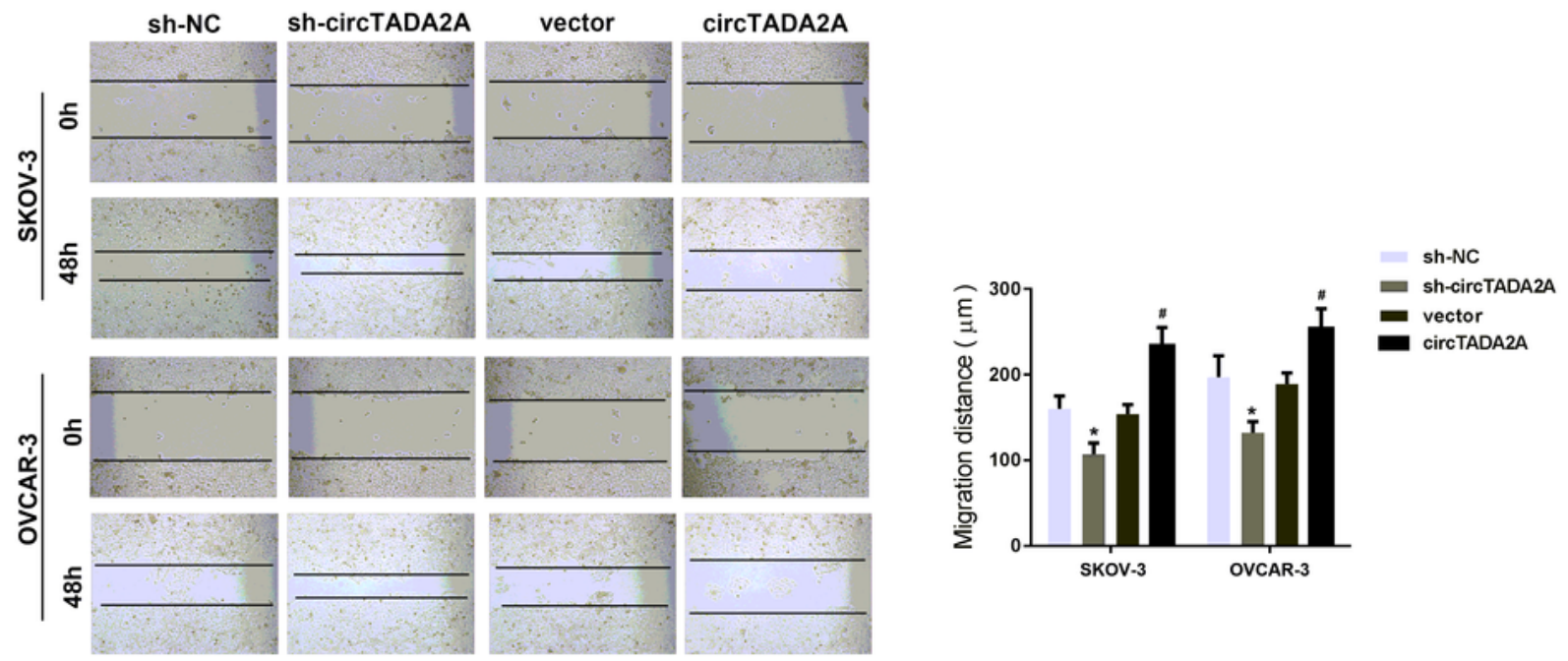

\section{Figure 2}

CircTADA2A promoted the proliferation and metastasis of OC cell. OC cells were transfected with overexpressing vector or shRNA for circTADA2A as well as their negative control respectively. (A) QPCR was used to evaluate the expression level of circTADA2A to evaluate the efficient of overexpressing vector or siRNA. (B) MTT was performed to detect the proliferation of OC cells. (C) Colony formation assay was performed to evaluate the colony formation ability of OC cell. (D) Transwell assay was used to 
investigate the migration of the $\mathrm{OC}$ cell. (E) Wound healing assay was carried out to evaluate the migration of $\mathrm{OC}$ cells. ${ }^{*} \mathrm{P}<0.05$, compared with the sh-NC group. $\mathrm{AP}<005$, compared with the vector group.

A

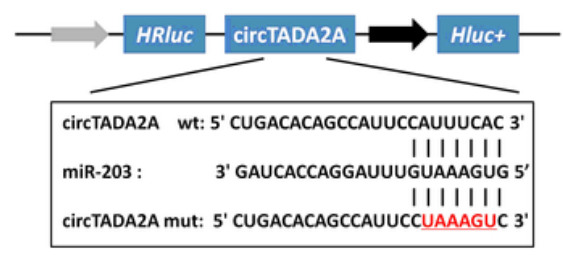

D

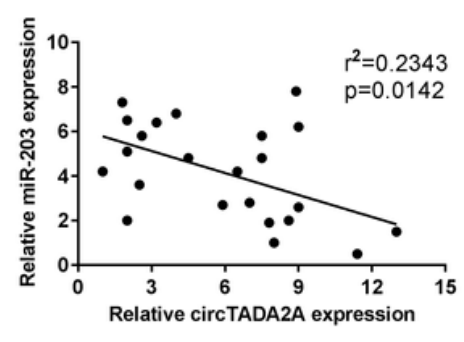

B

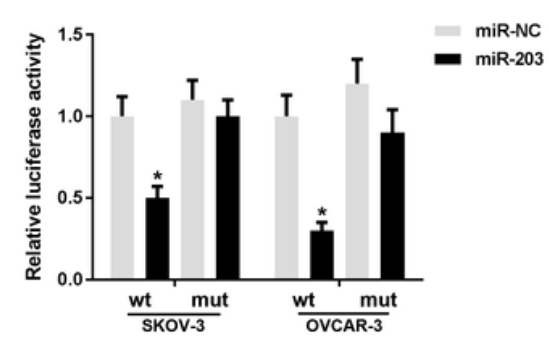

E

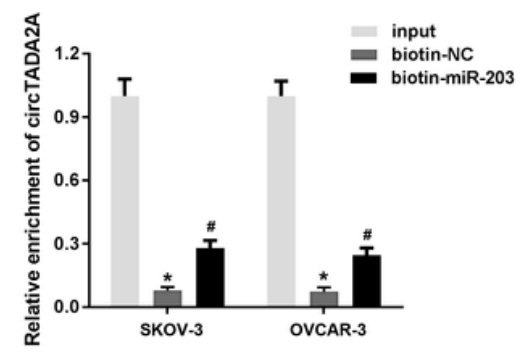

C
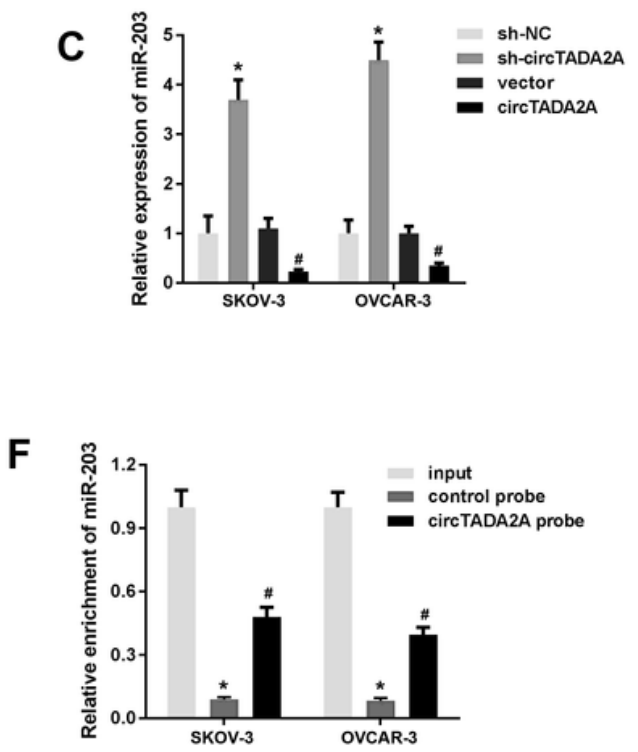

\section{Figure 3}

CircTADA2A directly targets miR-203 in OC cell. (A) The seed sequence of circTADA2A that binds to miR203 was showed, the sequences precisely modified were marked in red. (B) Luciferase activity assay was carried out in OC cell transfected with miR-203 mimic and the mimic control as well as the reporter vector containing wild type or mutant binding sequence of circTADA2A. (C) The level of miR-203 after different treatment was evaluated using GPCR. (D) Pearson analysis was performed to investigate the relation between circTADA2A and miR-203. (E, F) RNA pull down was carried out using specific probe for circTADA2A and miR-203 respectively labeled by biotin. ${ }^{*} \mathrm{P}<0.05$, compared with the miR-NC, sh-NC and input group. $\mathrm{AP}<005$, compared with the bio-NC and control probe group. 
A

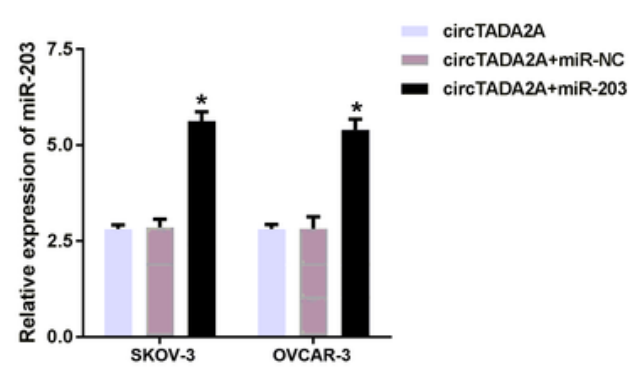

B

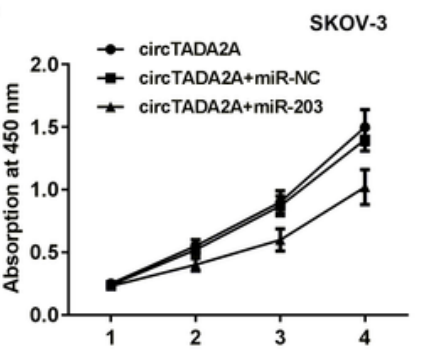

OVCAR-3

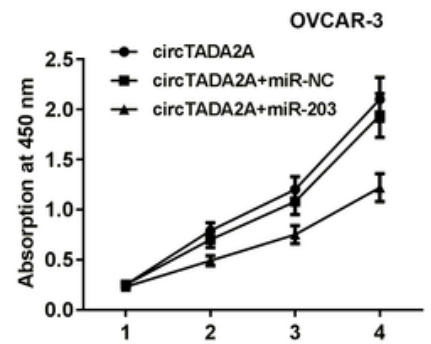

C
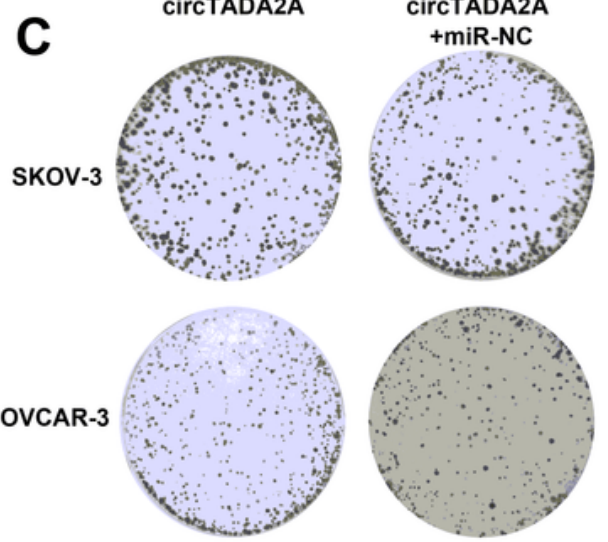

D
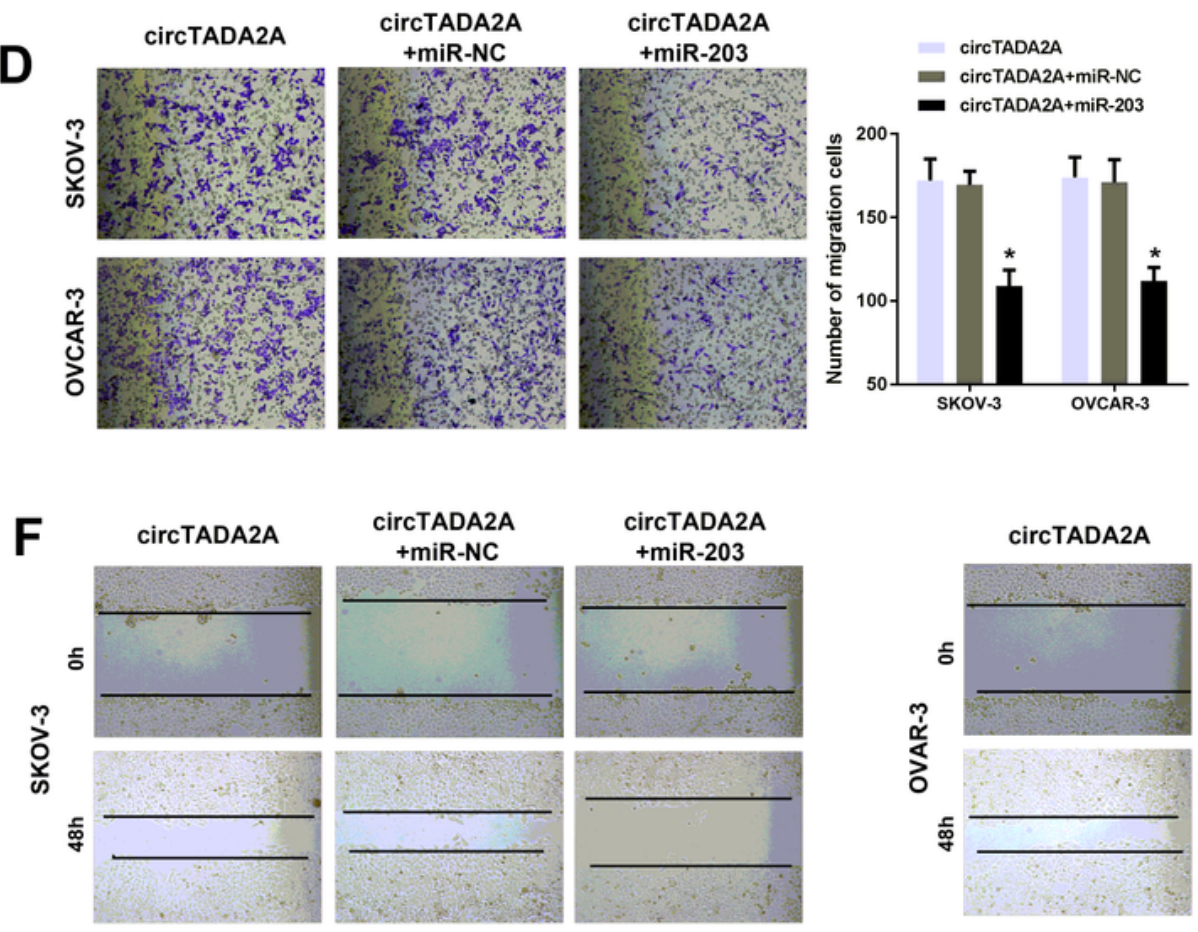

$\mathbf{E}$
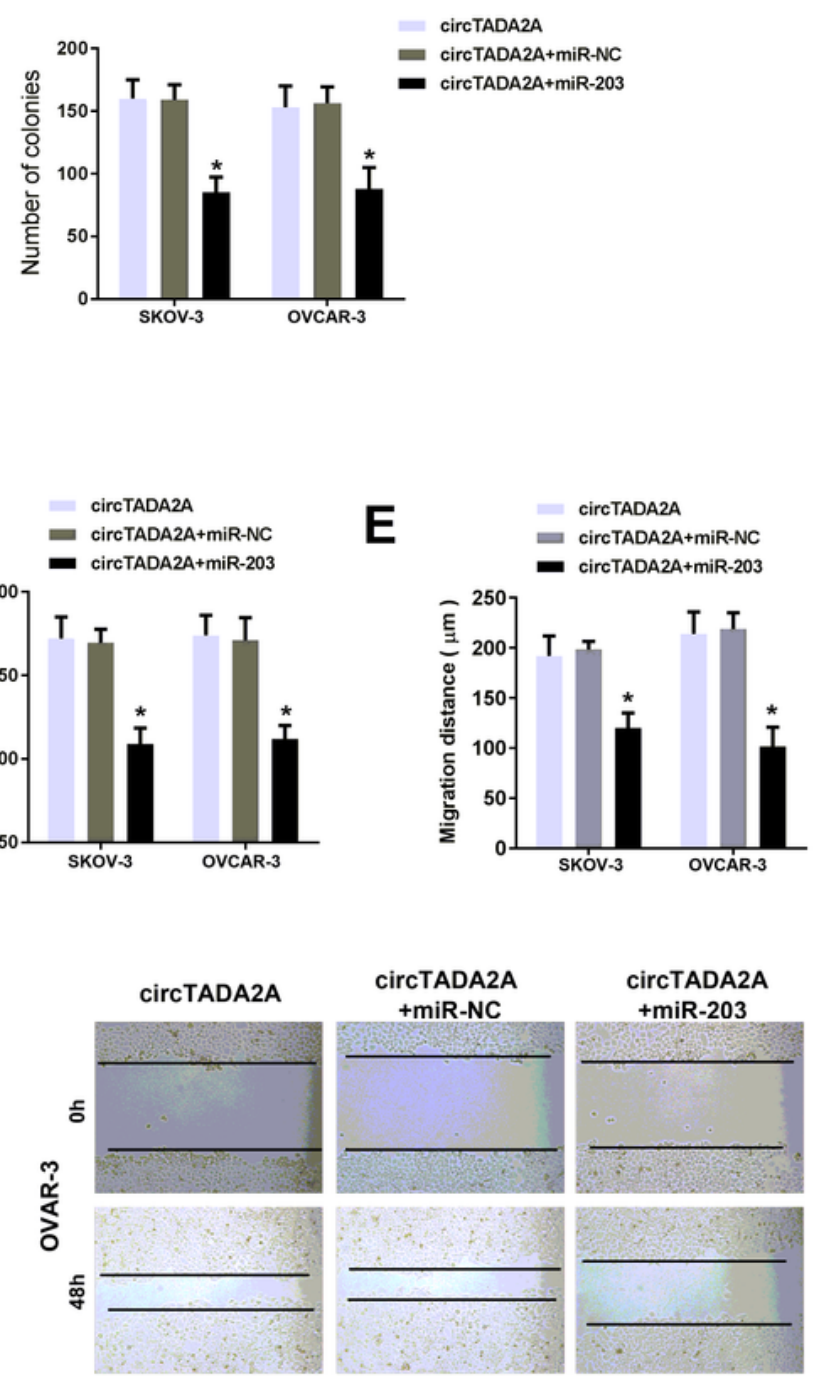

Figure 4

MiR-203 reversed the effect of circTADA2A on promoing the proliferation and metastasis of OC cell. OC cells were transfected with overexpressing vector for circTADA2A as well as miR-203 along with the negative control respectively. (A) QPCR was used to evaluate the expression level of miR-203 to confirm the influence of circTADA2A overexpression on the level of miR-203. (B) MTT was performed to detect the proliferation of OC cells. (C) Colony formation assay was performed to evaluate the colony formation 
ability of OC cell. (D) Transwell assay was used to investigate the migration of the OC cell. (E, F) Wound healing assay was carried out to evaluate the migration of $\mathrm{OC}$ cells. ${ }^{*} \mathrm{P}<005$, compared with the circTADA2A+miR-NC group.

A

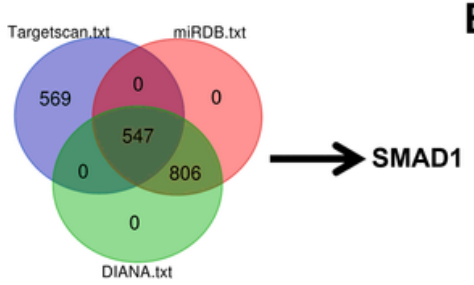

D

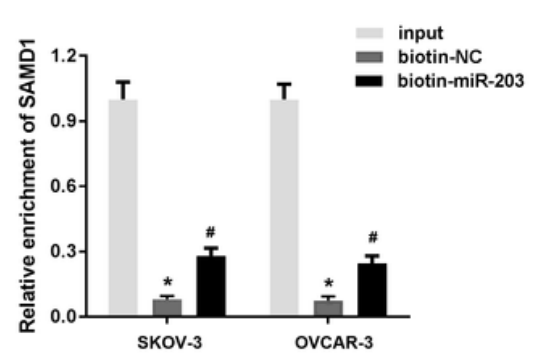

G

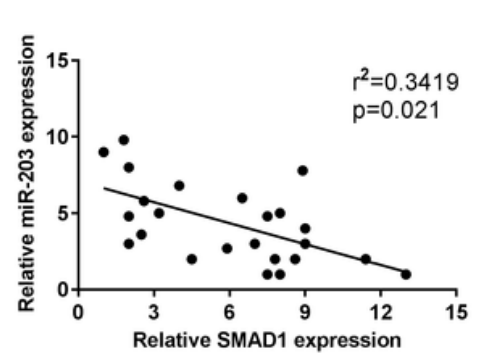

B

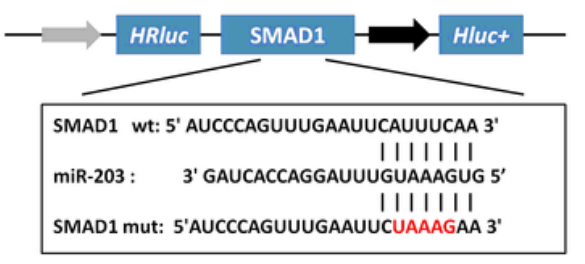

E

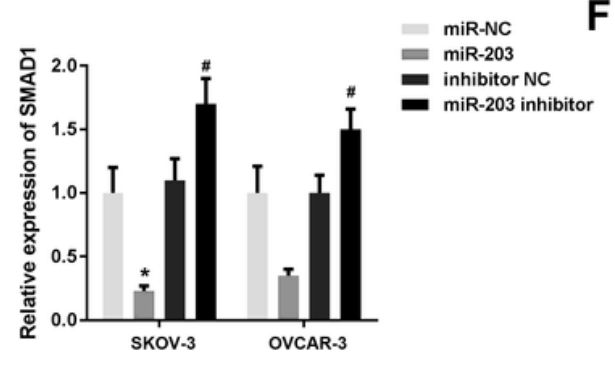

H

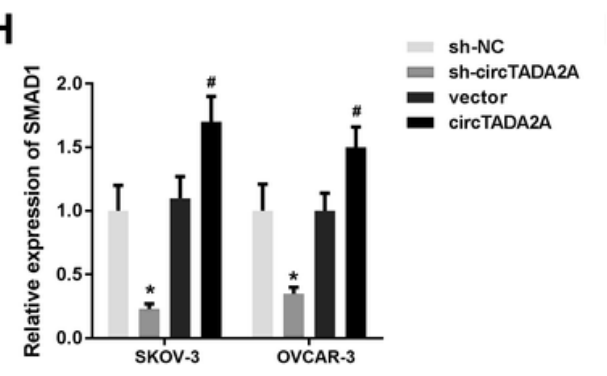

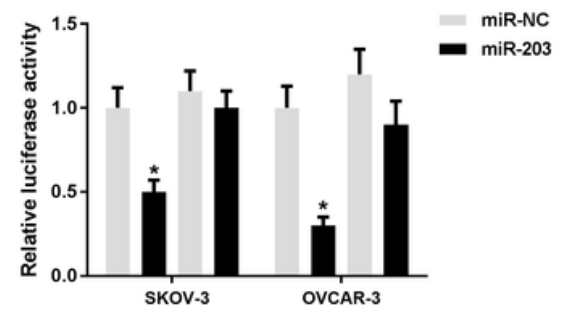

$\mathbf{F}$

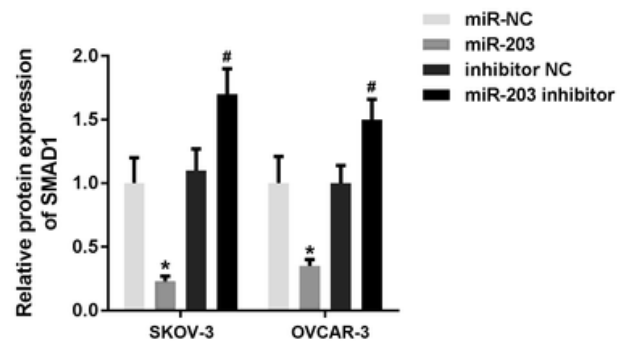

I

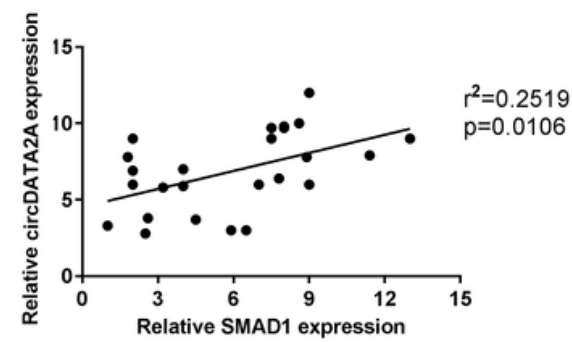

Figure 5

MiR-203 directly targets SMAD1 in OC cell. (A) Bioinformatics analysis including Targetscan 7.2, miRDB and DIANA were carried out to predict the target gene of miR-203. (B) The seed sequence of miR-203 that binds to SMAD1 was showed, the sequences precisely modified were marked in red. (C) Luciferase activity assay was carried out in OC cell transfected with miR-203 mimic and the mimic control as well as the reporter vector containing wild type or mutant binding sequence of SMAD1. (D) RNA pull down using specific probe was carried out to detect the interacting gene of miR-203. (E, F) The level of miR-203 after different treatment was evaluated using qPCR. (G-I) Pearson analysis was performed to investigate the relation between circTADA2A and miR-203 and also between miR-230 and SMAD1. *P $<0.05$, compared with the miR-NC, sh-NC and input group. $\# \mathrm{P}<005$, compared with the inhibitor $\mathrm{NC}$ and vector group. 
A

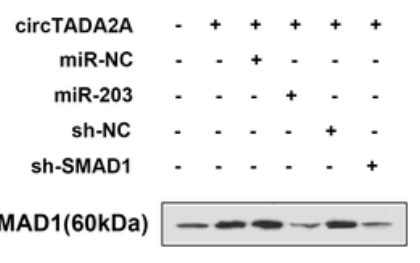

$\mathrm{N}$-cadherin (100kDa) ----

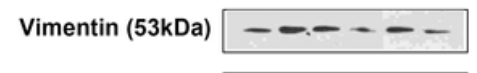

MMP9 (84kDa) $-\infty--$

E-cadherin $(97 \mathrm{kDa}) \longrightarrow-\ldots$

p-AKT(56kDa) $\longrightarrow-\longrightarrow-$

AKT(56kDa) $-\cdots-$

p-GSK3ß(47kDa) $\cdots---$

GSK3ß(47kDa) -----

GAPDH (37kDa)

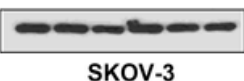

B

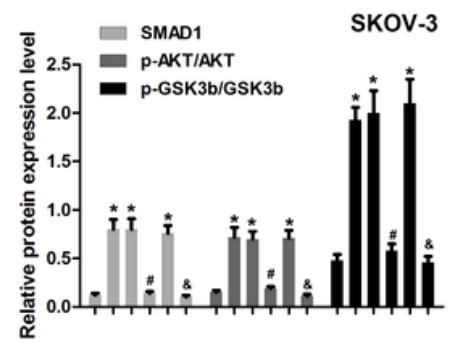

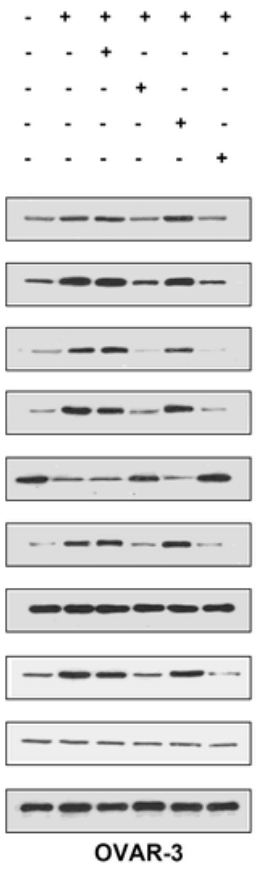

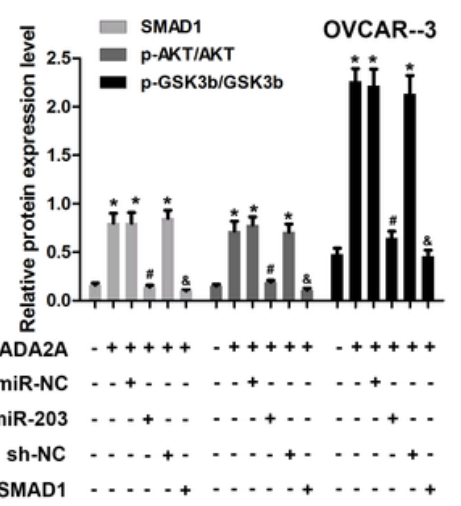

SKoV-3

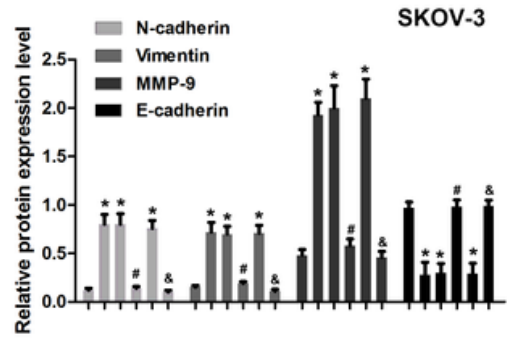

OVCAR-3

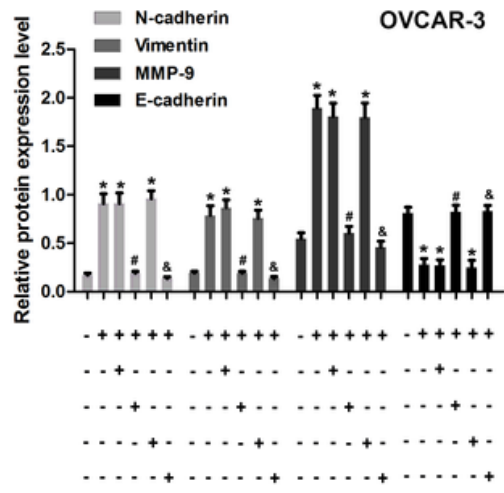

\section{Figure 6}

Circ-TADA2A involved in the regulatory of AKT/GSK3 and EMT proteins expression. (A, B) Western blot was used to detect the expression level of AKT/GSK3 and EMT proteins. ${ }^{*} P<0.05$, compared with the control group, \#P<0.05, compared with the circTADA2A+miR-NC group, \&P<0.05, compared with the circTADA2A+sh-NC group. 
A

\section{sh-circTADA2A}

sh-NC

circTADA2A
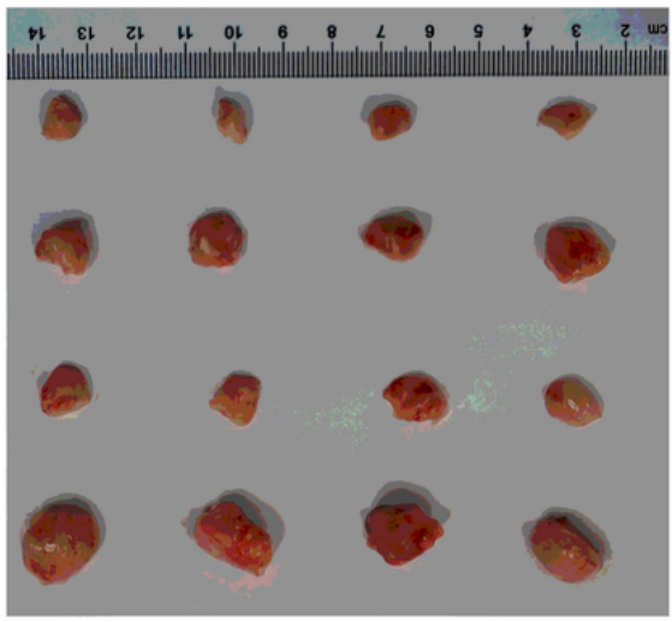

B

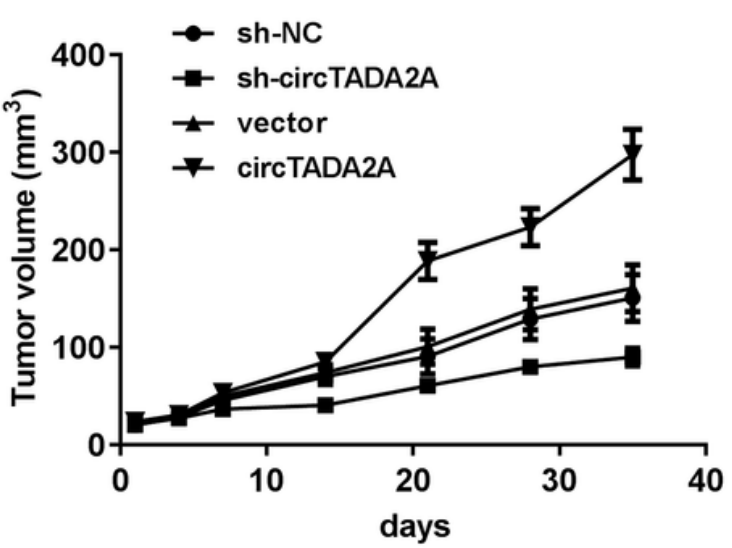

C

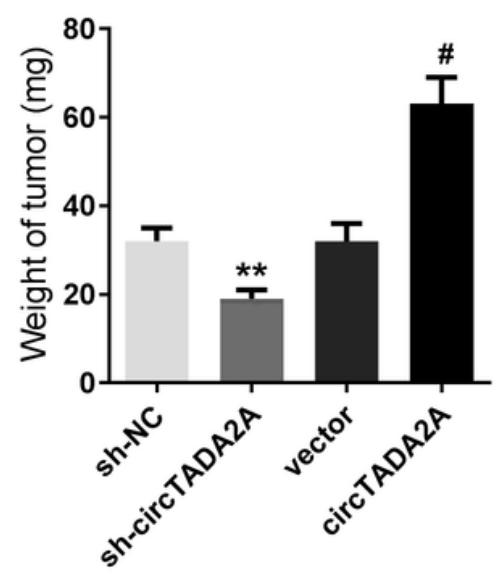

D

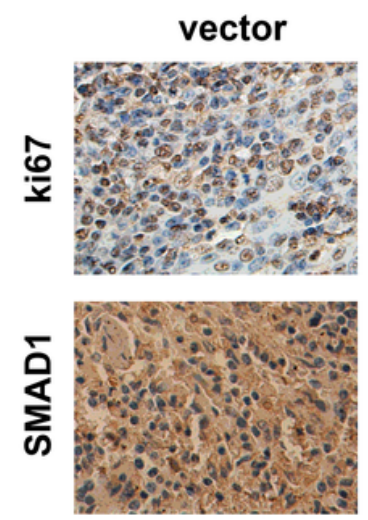

circTADA2A
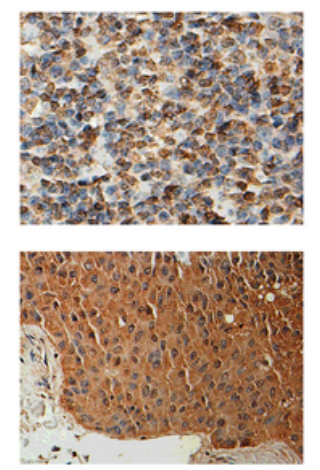

sh-NC
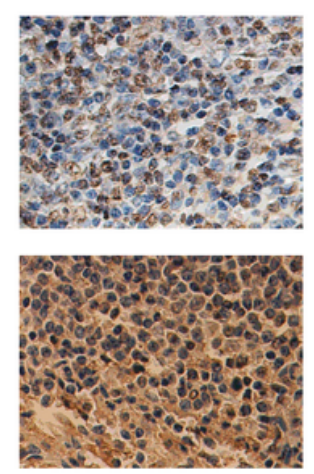

sh-circTADA2A
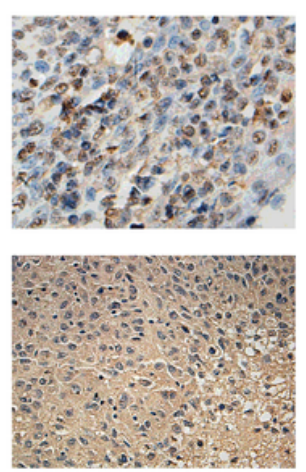

Figure 7

CircTADA2A promoted the growth of OC cells in vivo. Xenograft tumor model was established by subcutaneously injecting SKOV-3 cells stably over-expressing or deletion circTADA2A as well as the negative control in the dorsal flank area of nude mice. (A) The picture of the nude mice and the tumors in each group. (B) The growth curve of the tumor in the different groups. (C) The weight of the tumors in each group was calculated. (D) IHC was used to detect the ki67 and SMAD1 expression $(\times 100)$. ${ }^{*}<0.05$, compared with the normal group. \# $<<0.01$, compared with the vector group. 